\title{
ANISOTROPIC YOUNG DIAGRAMS AND JACK SYMMETRIC FUNCTIONS
}

\author{
S. KEROV
}

\begin{abstract}
We study the Young graph with edge multiplicities $\varkappa_{\alpha}(\lambda, \Lambda)$ arising in a Pieritype formula $p_{1}(x) P_{\lambda}(x ; \alpha)=\sum_{\Lambda: \lambda \nearrow \Lambda} \varkappa_{\alpha}(\lambda, \Lambda) P_{\Lambda}(x ; \alpha)$ for Jack symmetric polynomials $P_{\lambda}(x ; \alpha)$ with a parameter $\alpha$. Starting with $\operatorname{dim}_{\alpha} \varnothing=1$, we define recurrently the numbers $\operatorname{dim}_{\alpha} \Lambda=\sum \varkappa_{\alpha}(\lambda, \Lambda) \operatorname{dim}_{\alpha} \lambda$, and we set $\varphi(\lambda)=\prod_{b \in \lambda}(a(b) \alpha+l(b)+1)^{-1}$ (where $a(b)$ and $l(b)$ are the arm- and leg-length of a box $b)$.

New proofs are given for two known results. The first is the $\alpha$-hook formula $\operatorname{dim}_{\alpha} \lambda=$ $n ! \alpha^{n} \prod_{b \in \lambda}((a(b)+1) \alpha+l(b))^{-1}$. Secondly, we prove (for all $u, v \in \mathbb{C}$ ) the summation formula $\sum_{\Lambda: \lambda \nearrow \Lambda}\left(c_{\alpha}(b)+u\right)\left(c_{\alpha}(b)+v\right) \varkappa_{\alpha}(\lambda, \Lambda) \varphi(\Lambda)=(n \alpha+u v) \varphi(\lambda)$, where $c_{\alpha}(b)$ is the $\alpha$-content of a new box $b=\Lambda \backslash \lambda$. If $\alpha=1$, this identity implies the existence of an interesting family of positive definite central functions on the infinite symmetric group.

The approach is based on the interpretation of a Young diagram as a pair of interlacing sequences, so that analytic techniques may be used to solve combinatorial problems. We show that when dealing with Jack polynomials $P_{\lambda}(x ; \alpha)$, it makes sense to consider anisotropic Young diagrams made of rectangular boxes of size $1 \times \alpha$.
\end{abstract}

Introduction. The basic observation behind this paper is that the analytic notion of a pair of interlacing sequences is a natural generalization of the combinatorial notion of Young diagram (see [5] for more details and [3], [4] for applications of combinatorial methods to the analysis of interlacing sequences). We show that simple analytic facts related to interlacing sequences imply the hook formulae for dimensions of (representations corresponding to) Young diagrams, and for transition probabilities of the Plancherel measure of the infinite symmetric group. More generally, we obtain transition probabilities for the family of $z$-measures introduced in [6] which forms a deformation of the Plancherel measure. Both results admit a straightforward generalization in which the branching of Schur functions is replaced with that of Jack symmetric polynomials.

The analytic approach to the hook formula for ordinary dimensions was the subject of the papers [1], [8], [11]. The point of this note is that the considerations of [1] provide,

Key words and phrases. Interlacing sequences, Young diagrams, Jack symmetric polynomials, $\alpha-$ hook formula, central Markov chains.

Partially supported by the Federal Grant Program "Integration", No. 326.53, and by MSRI at Berkeley.

Typeset by $\mathcal{A} \mathcal{M S}-\mathrm{TEX}_{\mathrm{E}}$ 
with no extra effort, similar facts for the dimensions related to Jack symmetric functions. The only difference is that we identify Young diagrams with interlacing sequences in another way, taking into account the parameter $\alpha$ of Jack polynomials. Roughly, the ordinary Young diagrams should be dilated by the factor of $\alpha$ along the horizontal axis. One can also say that the new "diagrams" are built with rectangle "boxes" of width $\alpha$ and unit height, instead of unit square boxes in case of ordinary Young diagrams. We call them anisotropic Young diagrams.

The paper is organized as follows. We start in Section 1 by recalling the identification of a Young diagram with a pair of interlacing sequences. In Section 2 we use partial fractions to associate with a pair of interlacing sequences two discrete probability distributions. As explained in Sections 3-4, these distributions generalize the transition and co-transition distributions

$$
p_{\lambda}(\Lambda)=\frac{\operatorname{dim} \Lambda}{|\Lambda| \operatorname{dim} \lambda}, \quad q_{\Lambda}(\lambda)=\frac{\operatorname{dim} \lambda}{\operatorname{dim} \Lambda} ; \quad \lambda \nearrow \Lambda
$$

defined in combinatorial terms. In Section 5 we compute the moments of the transition distribution assocoated with a pair of interlacing sequences and show that the first three moments depend not on the diagram itself but only on its number of boxes. In Section 6 we give a short analytic proof of the $\alpha$-hook formula first found by Stanley in [10]. In the final Section 7 we establish our second main result, Theorem 7.5. It generalizes to the case of Jack symmetric polynomials the family of central measures on the Young lattice (or, equivalently, the family of spherical functions on the infinite symmetric group) introduced in [6].

Acknowledgement. I appreciate very much indeed the numerous discussions I had with G. I. Olshanskii on the relationship between combinatorics and analysis of Young diagrams, and on the combinatorics of Jack polynomials in particular. I am also indebted to S. Fomin, A. Okounkov and A. Vershik for their interest in this piece of work. The paper was completed during my visit to MSRI, Berkeley. It is my pleasure to thank the organizers of the Combinatorics 1997 Program for the invitation and financial support.

1. Interlacing sequences and Young diagrams. The aim of this Section is to explain that Young diagrams can be naturally regarded as integer interlacing sequences.

Given a finite set $\left\{x_{1}, \ldots, x_{d}\right\}$ of distinct real numbers, we shall always enumerate its elements in the increasing order, and identify the set with the corresponding sequence.

(1.1) Definition. Two sets (or increasing sequences) $y_{1}, \ldots, y_{d-1}$ and $x_{1}, \ldots, x_{d-1}, x_{d}$ are said to be interlacing, iff

$$
x_{1}<y_{1}<x_{2}<\ldots<x_{d-1}<y_{d-1}<x_{d} .
$$

The number $c=\sum x_{k}-\sum y_{k}$ is called the center of interlacing sequences. 
With every pair of interlacing sequences (1.2) we associate a piecewise linear continuous function $v=\omega(u)$, such that

(i) $\quad \omega^{\prime}(u)=+1, \quad$ if $x_{k}<u<y_{k}, \quad k=1, \ldots, d-1$;

(ii) $\quad \omega^{\prime}(u)=-1, \quad$ if $y_{k}<u<x_{k+1}, \quad k=1, \ldots, d-1$;

(iii) $\quad \omega(u)=|u-c|, \quad$ if $u<x_{1}$ or $u>x_{d}$.

One can easily see that such a function exists, and is uniquely determined by the properties (1.3). In fact, it follows from (iii), that $\omega\left(x_{1}\right)=c-x_{1}$ and $\omega\left(x_{d}\right)=x_{d}-c$. By (i) and (ii) we know that $\omega\left(y_{k}\right)-\omega\left(x_{k}\right)=y_{k}-x_{k}$ and $\omega\left(x_{k+1}\right)-\omega\left(y_{k}\right)=y_{k}-x_{k+1}$. This implies that

$$
\begin{aligned}
& \omega\left(x_{k}\right)=\sum_{i=1}^{k-1}\left(y_{i}-x_{i}\right)+\sum_{j=k}^{d-1}\left(x_{j+1}-y_{j}\right), \\
& \omega\left(y_{k}\right)=\sum_{i=1}^{k}\left(y_{i}-x_{i}\right)+\sum_{j=k}^{d-1}\left(x_{j+1}-y_{j}\right) .
\end{aligned}
$$

The region

$$
S_{\omega}=\left\{(u, v) \in \mathbb{R}^{2}:|u-c| \leq v<\omega(u)\right\}
$$

between the graphs of functions $v=\omega(u)$ and $v=|u-c|$ resembles the shape of a Young diagram, see Fig. 1. We say that $\omega$ is the diagram of interlacing sequences (1.2), and that the region (1.5) is its shape. We define the area of this shape as

$$
A(\omega)=\sum_{i<j}\left(y_{i}-x_{i}\right)\left(x_{j}-y_{j-1}\right) .
$$

We denote by $\mathbb{I}_{d}$ the space of interlacing sequences (1.2) with $2 d-1$ entries, and by $\mathbb{I}=\bigcup \mathbb{I}_{d}$ the set of all interlacing sequences. The topology of the space $\mathbb{I}$ is that of uniform convergence of associated diagrams.
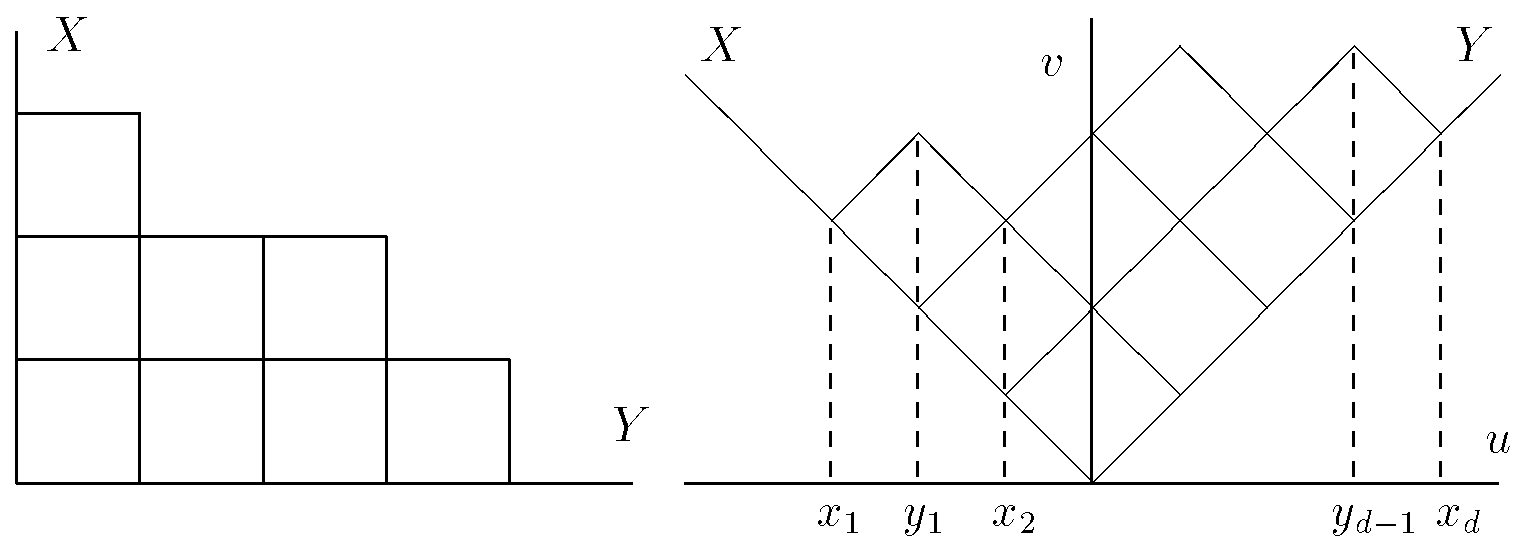
Fig. 1. A Young diagram as a pair of interlacing sequences.

The shape of a true Young diagram $\lambda=\left(\lambda_{1}, \ldots, \lambda_{m}\right)$ is uniquely determined by the contents $y_{1}, \ldots, y_{d-1}$ of its corner boxes, and the contents $x_{1}, \ldots, x_{d-1}, x_{d}$ of corner boxes of the compliment of $\lambda$ in $\mathbb{R}^{2}$. (Recall that the content of a box $b=(i, j)$ on the crossing of the $i$-th row and $j$-th column is defined as $c(b)=j-i$. It will also be convenient to say that each point in the plane with the coordinates $(u, v)$ has the content $c(u, v)=v-u$. Then the content of a box coincides with that of its center.) The sequences of integers $y_{1}, \ldots, y_{d-1}$ and $x_{1}, \ldots, x_{d-1}, x_{d}$ interlace. Also, the center of such a pair is always zero, $c=\sum x_{k}-\sum y_{k}=0$.

In the opposite direction, every pair of interlacing sequences (1.2) with integer entries and zero center represents a true Young diagram. The number of rows $m$ in this diagram equals $-x_{1}$, and $\lambda_{1}=x_{d}$ is the length of the first row. The number of boxes $|\lambda|=A(\omega)$ equals the area of the associated diagram $\omega$.

Therefore, the set $\mathbb{Y}$ of Young diagrams can be regarded as the lattice of integer points in the space $\mathbb{I}_{0}$ of interlacing sequences with zero center.

Conventionally, one draws a Young diagram $\lambda$ with horizontal rows in decreasing order, so that the graph of the associated piecewise linear function should be rotated by $45^{\circ}$. The contents of the points of the horizontal parts on the border of $\lambda$ form the intervals $\left(x_{i}, y_{i}\right)$, and those of the vertical parts - the intervals $\left(y_{i}, x_{i+1}\right), i=1, \ldots, d-1$.

2. Transition and co-transition distributions of an interlacing sequence. In this Section we associate with every pair of interlacing sequences two discrete probability distributions. In the particular case of sequences corresponding to a Young diagram, the distributions coincide with the transition and co-transition distributions for the Plancherel measure of the infinite symmetric group. All the facts that we recall here are well known, though we use the combinatorial terminology related to the Young lattice.

Given a pair (1.2) of sets $X=\left(x_{1}, \ldots, x_{d-1}, x_{d}\right)$ and $Y=\left(y_{1}, \ldots, y_{d-1}\right)$, we denote by $P(u)=\prod\left(u-x_{k}\right)$ and $Q(u)=\prod\left(u-y_{k}\right)$ the monic polynomials with corresponding roots. Let us expand the rational fraction $R(u)=Q(u) / P(u)$ as a sum of partial fractions,

$$
\frac{\left(u-y_{1}\right) \ldots\left(u-y_{d-1}\right)}{\left(u-x_{1}\right) \ldots\left(u-x_{d-1}\right)\left(u-x_{d}\right)}=\sum_{k=1}^{d} \frac{\mu_{k}}{u-x_{k}} .
$$

Multiplying both sides by $u$ and taking the limit $u \rightarrow \infty$ we observe that

$$
\sum_{k=1}^{d} \mu_{k}=1
$$

(2.2) Lemma. The following two properties of the fraction (2.1) are equivalent:

$$
x_{1}<y_{1}<x_{2}<\ldots<x_{d-1}<y_{d-1}<x_{d} \quad \Longleftrightarrow \quad \mu_{1}>0, \ldots, \mu_{d}>0 .
$$


Proof. By the residue formula,

$$
\mu_{k}=\frac{Q\left(x_{k}\right)}{P^{\prime}\left(x_{k}\right)}=\prod_{i<k} \frac{x_{k}-y_{i}}{x_{k}-x_{i}} \prod_{j>k} \frac{x_{k}-y_{j-1}}{x_{k}-x_{j}} .
$$

Clearly, if the roots and poles of the fraction $R(u)$ interlace, then all the coefficients $\mu_{k}$ are positive.

Assume now that $\mu_{k}>0$ for all $k=1,2, \ldots, d$. Then the number of $y$-points to the right of the largest $x$-point $x_{d}$ is even. Moreover, it is zero. Otherwise, we could find two neighboring $x$-points, not separated by a $y$-point, and the corresponding coefficients $\mu$ would have opposite signs. A similar argument proves that there is the same number of $x$-points and $y$-points to the right of every $x_{k}$, so that the sequences interlace.

(2.3) Definition. To every pair of interlacing sequences $x_{1}<y_{1}<\ldots<y_{d-1}<x_{d}$ there corresponds a discrete probability distribution $\mu$ with the weights

$$
\mu_{k}=\prod_{i=1}^{k-1} \frac{x_{k}-y_{i}}{x_{k}-x_{i}} \prod_{j=k+1}^{d} \frac{x_{k}-y_{j-1}}{x_{k}-x_{j}}
$$

at the points $x_{k}, k=1,2, \ldots, d$. We refer to $\mu$ as to the transition distribution of the pair, and we call the weights $\mu_{k}$ its transition probabilities.

Another distribution associated with a pair of interlacing sequences arises from the decomposition

$$
\frac{\left(u-x_{1}\right) \ldots\left(u-x_{d-1}\right)\left(u-x_{d}\right)}{\left(u-y_{1}\right) \ldots\left(u-y_{d-1}\right)}=u-c-\sum_{k=1}^{d-1} \frac{\nu_{k}}{u-y_{k}} .
$$

(2.6) Lemma. The coefficient $c=c(\omega)$ in (2.5) coincides with the center of the diagram $\omega$, and the sum of the coefficients $\sum \nu_{k}=A(\omega)$ equals the area of its shape. The following two conditions are equivalent:

$$
x_{1}<y_{1}<x_{2}<\ldots<x_{d-1}<y_{d-1}<x_{d} \quad \Longleftrightarrow \quad \nu_{1}>0, \ldots, \nu_{d-1}>0 .
$$

Proof. In order to prove the first two claims of the Lemma we multiply both sides of (2.5) by the polynomial $P(u)$ and compare a number of first coefficients. Let $e_{k}(Y)$ and

$$
e_{k}(X)=\sum_{i_{1}<\ldots<i_{k}} x_{i_{1}} \ldots x_{i_{k}}
$$

denote the $k$-th elementary symmetric functions in the variables $Y=\left(y_{1}, \ldots, y_{d-1}\right)$ and $X=\left(x_{1}, x_{2}, \ldots, x_{d}\right)$ correspondingly. Up to the terms of degree $d-3$ and smaller,

$$
\begin{gathered}
u^{d}-e_{1}(X) u^{d-1}+e_{2}(X) u^{d-2}-\ldots=-u^{d-2} \sum \nu_{k}+\ldots \\
+(u-c)\left(u^{d-1}-e_{1}(Y) u^{d-2}+e_{2}(Y) u^{d-3}-\ldots\right),
\end{gathered}
$$


so that $c=e_{1}(X)-e_{1}(Y)$ and $\sum \nu_{k}=-e_{2}(X)+e_{1}(X) e_{1}(Y)-e_{1}^{2}(Y)+e_{2}(Y)=A(\omega)$.

By the residue formula,

$$
\nu_{k}=-\frac{P\left(y_{k}\right)}{Q^{\prime}\left(y_{k}\right)}=-\prod_{i}\left(y_{k}-x_{i}\right) \prod_{j \neq k}\left(y_{k}-y_{j}\right)^{-1}
$$

If the sequences $X$ and $Y$ interlace, the number of $x$-points to the right of each $y_{k}$ is one bigger than the number of $y$-points to the right of $y_{k}$. It follows that the coefficients $\nu_{k}$ are all positive.

Denote by $x(k), y(k)$ the numbers of $x$-points and $y$-points bigger than $y_{k}$. If the coefficient $\nu_{k}$ is positive, the difference $x(k)-y(k)$ is odd. In fact, it can not be negative. Otherwise, the biggest element would be $y_{d-1}$ or else we could find two $y$-points not separated by an $x$-point. In both cases some coefficient $\nu_{m}, m>k$, would be negative. The assumption $x(k)-y(k)>1$ also implies a contradiction, for there would be too many $y$-points to the left of $y_{k}$, and the sign of $\nu_{j}$ for one of these points would be negative. Hence, $x(k)=y(k)+1$ for all $k=1, \ldots, d-1$ which means that the sequences $X, Y$ interlace.

(2.8) Definition. Let $A=A(\omega)$ be the area (1.6) of the diagram $\omega$ associated with a pair of interlacing sequences (1.2). Denote by $\nu$ the system of weights

$$
\frac{\nu_{k}}{A}=\frac{\left(x_{d}-y_{k}\right)\left(y_{k}-x_{1}\right)}{A} \prod_{i=1}^{k-1} \frac{y_{k}-x_{i+1}}{y_{k}-y_{i}} \prod_{j=k+1}^{d-1} \frac{y_{k}-x_{j}}{y_{k}-y_{j}}
$$

at the points $y_{k}, k=1, \ldots, d-1$. By Lemma 2.6, $\nu$ is a probability distribution. We call $\nu$ the co-transition distribution, and the weights $\nu_{k} / A$ the co-transition probabilities of the pair.

3. Co-transition distribution of a Young diagram. We show in this Section that the transition and co-transition distributions introduced in Section 2 generalize the corresponding combinatorial notions. We start by recalling the definition of the Plancherel growth process (see [2] and [12] for more details) and the related combinatorial definitions of transition and co-transition probabilities.

We denote by $\mathbb{Y}_{n}$ the set of Young diagrams with $n$ boxes, and by $\mathbb{Y}=\bigcup \mathbb{Y}_{n}$ the lattice of all Young diagrams ordered by inclusion. We write $\lambda \nearrow \Lambda$, if the diagram $\Lambda$ covers $\lambda$ in $\mathbb{Y}$, i.e., if $\Lambda=\lambda \bigcup b_{0}$ is a union of $\lambda$ and an extra box $b_{0}$.

Let $\operatorname{dim} \lambda$ denote the number of standard Young tableaux of shape $\lambda \in \mathbb{Y}$. The dimension function $\operatorname{dim} \lambda$ may be characterized by the initial condition $\operatorname{dim} \varnothing=1$, and by the recurrence relation

$$
\operatorname{dim} \Lambda=\sum_{\lambda: \lambda \nearrow \Lambda} \operatorname{dim} \lambda
$$


(similar to that of the Pascal triangle) which can also be written as

$$
\sum_{\lambda: \lambda \nearrow \Lambda} \frac{\operatorname{dim} \lambda}{\operatorname{dim} \Lambda}=1
$$

The ratios $q_{\Lambda}(\lambda)=\operatorname{dim} \lambda / \operatorname{dim} \Lambda$ are called co-transition probabilities of the diagram $\Lambda$.

The celebrated hook formula for the dimension reads

$$
\operatorname{dim} \lambda=n ! \prod_{b \in \lambda} h^{-1}(b)
$$

where $h(b)=\left(\lambda_{i}-j\right)+\left(\lambda_{j}^{\prime}-i\right)+1$ is the hook length of the box $b=(i, j)$ on the crossing of $i$-th row and $j$-th column.

In order to prove (3.2), one can show that the right hand side enjoys a recurrence relation similar to (3.1). We shall derive this fact from Lemma 2.6. To this end, we first check that the combinatorial definition of co-transition probabilities is equivalent to the analytic one of Section 2.

(3.3) Lemma. Denote by $x_{1}<y_{1}<\ldots<y_{d-1}<x_{d}$ the interlacing sequences corresponding to a Young diagram $\Lambda$ with $A$ boxes, and let $\lambda$ be the Young diagram obtained from $\Lambda$ by removing a box with the content $y_{k}$. Let $f_{\lambda}$ denote the right hand side of (3.2). Then

$$
\frac{f_{\lambda}}{f_{\Lambda}}=\frac{\left(x_{d}-y_{k}\right)\left(y_{k}-x_{1}\right)}{A} \prod_{i=1}^{k-1} \frac{y_{k}-x_{i+1}}{y_{k}-y_{i}} \prod_{j=k+1}^{d-1} \frac{y_{k}-x_{j}}{y_{k}-y_{j}}
$$

Proof. By definition, the ratio in the left hand side can be written as

$$
\frac{f_{\lambda}}{f_{\Lambda}}=\frac{1}{|\lambda|} \prod_{b} \frac{h(b)+1}{h(b)}
$$

where the product runs over the boxes in the column of $\lambda$ above the new box $b_{0}=\Lambda \backslash \lambda$, and along the row of $\lambda$ to the left of $b_{0}$. The hook lengths $h(b)$ are taken with respect to the smaller diagram $\lambda$. Consider the intersection $I$ of the column with a block of equal rows of Young diagram $\lambda$, see Fig. 2a. The block corresponds to a vertical interval at the border of the diagram, and we denote by $y_{j}, x_{j+1}$ the contents of its endpoints. If $j>k$, the product along $I$ simplifies to

$$
\prod_{b \in I} \frac{h(b)+1}{h(b)}=\frac{\left(y_{j}-y_{k}+1\right)}{\left(y_{j}-y_{k}\right)} \frac{\left(y_{j}-y_{k}+2\right)}{\left(y_{j}-y_{k}+1\right)} \ldots \frac{\left(x_{j+1}-y_{k}\right)}{\left(x_{j+1}-y_{k}-1\right)}=\frac{\left(x_{j+1}-y_{k}\right)}{\left(y_{j}-y_{k}\right)}
$$

If $j=k$, the smallest hook of $I$ equals 1 , and the product reduces to $\prod_{I}(h+1) / h=$ $\left(x_{k+1}-y_{k}\right)$. 
In a similar way we consider the case where $I$ is the intersection of the row containing the new box $b_{0}$ with a block of equal columns of $\lambda$, see Fig. $2 \mathrm{~b}$. Such a block corresponds to a horizontal interval on the border of $\lambda$, and we denote by $x_{i}, y_{i}$ the contents of its endpoints. The product along $I$ reduces in this case to

$$
\prod_{b \in I} \frac{h(b)+1}{h(b)}=\frac{\left(y_{k}-y_{i}+1\right)}{\left(y_{k}-y_{i}\right)} \frac{\left(y_{k}-y_{i}+2\right)}{\left(y_{k}-y_{i}+1\right)} \cdots \frac{\left(y_{k}-x_{i}\right)}{\left(y_{k}-x_{i}-1\right)}=\frac{\left(y_{k}-x_{i}\right)}{\left(y_{k}-y_{i}\right)}
$$

if $i<k$, and to $\prod_{I}(h+1) / h=\left(y_{k}-x_{k}\right)$ if $i=k$.

The hook formula (3.2) readily follows from Lemmas (2.6) and (3.3). In fact, these results imply that $\sum f_{\lambda} / f_{\Lambda}=1$, hence the numbers $f_{\lambda}$ satisfy the same recurrence relations (3.1) as $\operatorname{dim} \lambda$ and the identity $\operatorname{dim} \lambda=f_{\lambda}$ follows.

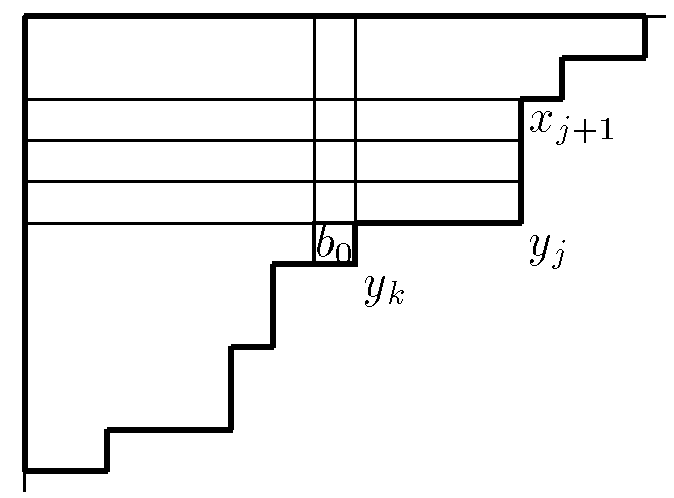

Fig. 2a

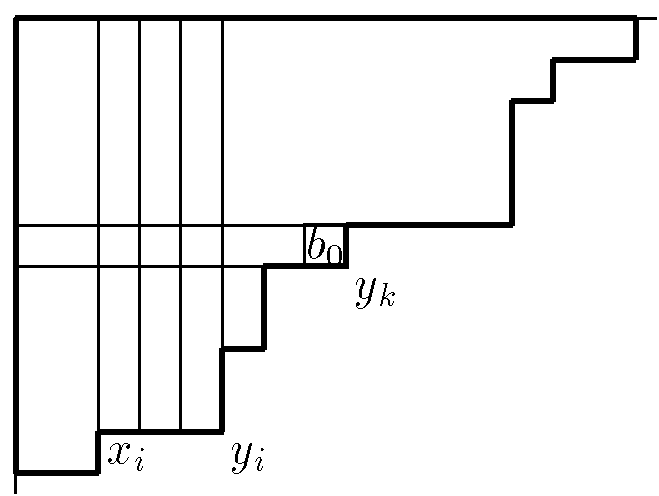

Fig. 2b

4. Plancherel transition distributions. Now we discuss the well known formula

$$
\sum_{\Lambda: \lambda \nearrow \Lambda} \operatorname{dim} \Lambda=(|\lambda|+1) \operatorname{dim} \lambda
$$

which has a number of different proofs. For instance, one can compute in two different ways the dimension of the representation Ind $\pi_{\lambda}$ of the symmetric group $\mathfrak{S}_{n+1}$ induced by the irreducible representation $\pi_{\lambda}$ (labeled by a Young diagram $\lambda$ ) of the subgroup $\mathfrak{S}_{n}$. A bijective proof can be obtained from the basic properties of the Robinson-Schensted insertion algorithm. Here we derive (4.1) from the fact that the weights (2.4) associated with a pair of interlacing sequences always form a probability distribution.

The formula (4.1) can be written as

$$
\sum_{\Lambda: \lambda \nearrow \Lambda} \frac{\operatorname{dim} \Lambda}{(n+1) \operatorname{dim} \lambda}=1
$$


so that the numbers $\mu_{\lambda}(\Lambda)=\operatorname{dim} \Lambda /(n+1) \operatorname{dim} \lambda$, where $\lambda \nearrow \Lambda$, can be taken as the weights of a discrete probability distribution $\mu_{\lambda}$ associated with a Young diagram $\lambda \in \mathbb{Y}_{n}$.

Consider the Markov chain on the Young lattice called the Plancherel growth process. By definition, it starts at the empty diagram $\varnothing$, and has the transition probabilities $p(\lambda, \Lambda)=\operatorname{dim} \Lambda /(n+1) \operatorname{dim} \lambda$. One can easily check that the probability to cross the $n$-th level $\mathbb{Y}_{n}$ of the Young lattice at a particular Young diagram $\lambda$ equals $M_{n}(\lambda)=\operatorname{dim}^{2} \lambda / n$ !, the weight of this diagram with respect to the Plancherel measure of the group $\mathfrak{S}_{n}$. In many ways, the chain can be regarded as the Plancherel measure of the infinite symmetric group $\mathfrak{S}_{\infty}$. Following [7], we call $\mu_{\lambda}$ Plancherel transition distribution.

The Plancherel growth process is a central Markov chain, meaning that the probability to reach a Young diagram $\lambda$ along a fixed path (i.e., Young tableaux) depends only on $\lambda$, not on the choice of the path. In our case this probability equals $\operatorname{dim} \lambda / n$ !.

Recall that the co-transition probability $q(\lambda, \Lambda)$ of a Markov chain on the Young lattice is defined as the conditional probability to pass through a vertex $\lambda$, assuming that the next vertex is $\Lambda$. For every central Markov chain the co-transition probability is $q(\lambda, \Lambda)=\operatorname{dim} \lambda / \operatorname{dim} \Lambda$. In fact, this latter formula characterizes central Markov chains.

(4.3) Lemma. Denote by $x_{1}<y_{1}<x_{2}<\ldots<y_{d-1}<x_{d}$ the interlacing sequences associated with a Young diagram $\lambda \in \mathbb{Y}_{n}$. Assume that a diagram $\Lambda$, where $\lambda \nearrow \Lambda$, is obtained from $\lambda$ by attaching a box $b_{0}$ with the content $x_{k}$. Then

$$
\frac{\operatorname{dim} \Lambda}{(n+1) \operatorname{dim} \lambda}=\prod_{i=1}^{k-1} \frac{x_{k}-y_{i}}{x_{k}-x_{i}} \prod_{j=k+1}^{d} \frac{x_{k}-y_{j-1}}{x_{k}-x_{j}}
$$

i.e., combinatorial and analytic definitions of transition probabilities are equivalent.

Proof. By the hook formula (3.2), the left hand side can be written as

$$
\frac{\operatorname{dim} \Lambda}{(n+1) \operatorname{dim} \lambda}=\prod_{b} \frac{h(b)}{h(b)+1}
$$

where the product runs over the boxes of $\lambda$ in the row, as well as in the column, containing the new box $b_{0}$. All the hook lengths $h(b)$ are taken with respect to the diagram $\lambda$.

The remaining argument is quite similar to that of the proof of Lemma 3.3. Consider, for instance, the intersection $I$ of the column through the box $b_{0}$ with a block of equal rows of $\lambda$. Let $y_{j-1}, x_{j}$ be the contents of the endpoints of the vertical interval on the crossing of these rows with the graph of the diagram $\lambda$. Then

$$
\prod_{b \in I} \frac{h(b)}{h(b)+1}=\frac{\left(y_{j-1}-x_{k}\right)}{\left(y_{j-1}-x_{k}+1\right)} \frac{\left(y_{j-1}-x_{k}+1\right)}{\left(y_{j-1}-x_{k}+2\right)} \ldots \frac{\left(x_{j}-x_{k}-1\right)}{\left(x_{j}-x_{k}\right)}=\frac{\left(x_{k}-y_{j-1}\right)}{\left(x_{k}-x_{j}\right)} .
$$


In a similar way we deal with the product along the part $I$ of the row through $b_{0}$, corresponding to a horizontal interval with the endpoints $x_{i}, y_{i}$ on the border of $\lambda$. It is equal to

$$
\prod_{b \in I} \frac{h(b)}{h(b)+1}=\frac{\left(x_{k}-y_{i}\right)}{\left(x_{k}-y_{i}+1\right)} \frac{\left(x_{k}-y_{i}+1\right)}{\left(x_{k}-y_{i}+2\right)} \ldots \frac{\left(x_{k}-x_{i}-1\right)}{\left(x_{k}-x_{i}\right)}=\frac{\left(x_{k}-y_{i}\right)}{\left(x_{k}-x_{i}\right)}
$$

and the Lemma follows.

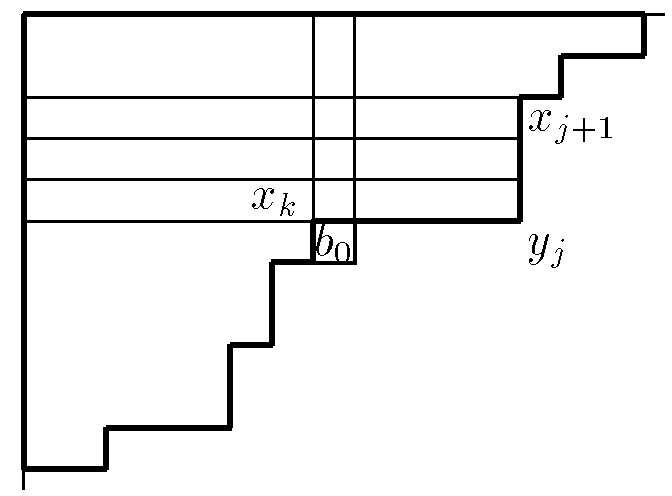

Fig. 3a

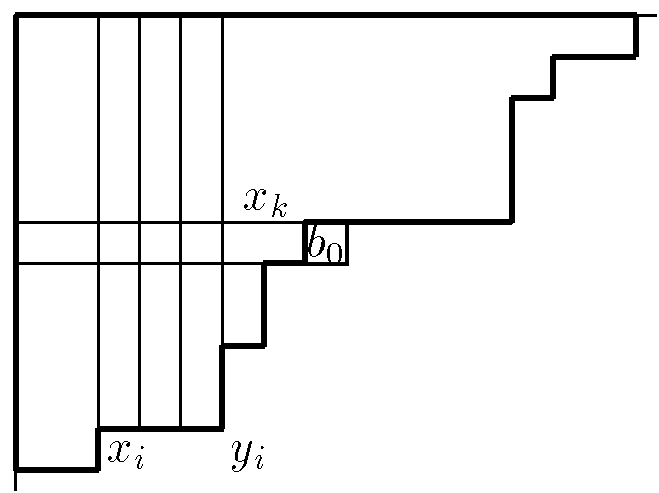

Fig. 3b

5. Transition distributions of $z$-measures. In this Section we find the moments of the transition distribution (2.4) of a pair of interlacing measures. Then we use these moments to define a family of central Markov chains on the Young lattice, introduced in $[6]$.

Let $\mu$ denote the transition distribution (2.4) of a pair $x_{1}<y_{1}<x_{2}<\ldots<x_{d}$ of interlacing sequences, and let

$$
h_{m}=\sum_{k=1}^{d} x_{k}^{m} \mu_{k}
$$

be the $m$-th moment of $\mu$. We consider the generating function

$$
R(u)=\sum_{m=0}^{\infty} \frac{h_{m}}{u^{m+1}}
$$

$$
\sum_{m=0}^{\infty} \frac{h_{m}}{u^{m+1}}=\frac{\left(u-y_{1}\right) \ldots\left(u-y_{d-1}\right)}{\left(u-x_{1}\right) \ldots\left(u-x_{d-1}\right)\left(u-x_{d}\right)}
$$


Proof. Since $1 /\left(u-x_{k}\right)=\sum_{m \geq 0} x_{k}^{m} / u^{m+1}$, we obtain that

$$
R(u)=\sum_{k=1}^{d} \frac{\mu_{k}}{u-x_{k}},
$$

and the claim follows from the identity (2.1).

(5.5) Corollary. Let $c$ and $A$ denote the center and the area of the diagram associated with interlacing sequences $(X, Y)$. Then the mean value of their transition distribution is $h_{1}=c$, and its variance is $h_{2}-h_{1}^{2}=A$.

Proof. Using the notations of Section 2 for the elementary symmetric functions, we derive from (5.4) that $h_{1}=e_{1}(X)-e_{1}(Y)=c$ and $h_{2}=e_{1}^{2}(X)-e_{2}(X)-e_{1}(X) e_{1}(Y)+$ $e_{2}(Y)$. Hence, $h_{2}-h_{1}^{2}=-e_{2}(X)+e_{1}(X) e_{1}(Y)+e_{2}(Y)-e_{1}^{2}(Y)=A$.

(5.6) Corollary. Given two complex parameters u, v, consider the numbers

$$
p_{u, v}(\lambda, \Lambda)=\frac{(c(b)+u)(c(b)+v)}{n+u v} \frac{\operatorname{dim} \Lambda}{(n+1) \operatorname{dim} \lambda},
$$

where $\lambda \in \mathbb{Y}_{n}, \lambda \nearrow \Lambda$ and $b=\Lambda \backslash \lambda$. Then

$$
\sum_{\Lambda: \lambda \nearrow \Lambda} p_{u, v}(\lambda, \Lambda)=1
$$

If $v=\bar{u}$, the numbers (5.7) are positive, and may be taken as transition probabilities of a Markov chain on the Young lattice. All these chains are central. In the limit $u \rightarrow \infty$ we obtain the Plancherel growth process, hence the family may be considered as a deformation of the Plancherel measure of the infinite symmetric group $\mathfrak{S}_{\infty}$.

6. Co-transition probabilities determined by the Jack symmetric functions. Following [9], Chapter VI.10, we denote by $P_{\lambda}(x)=P_{\lambda}\left(x_{1}, x_{2}, \ldots ; \alpha\right) ; \lambda \in \mathbb{Y}$, the family of Jack symmetric functions with the parameter $\alpha$. For a fixed value of $\alpha$ the functions $P_{\lambda}$ form a linear basis in the symmetric function algebra.

Denote by $p_{1}(x)=x_{1}+x_{2}+\ldots$ the sum of variables, and consider the decomposition of the product $p_{1} P_{\lambda}$ in the basis $P_{\Lambda}$. It is known ([9], VI.6.24(iv) and V.10.10) that

$$
p_{1}(x) P_{\lambda}(x ; \alpha)=\sum_{\Lambda: \lambda \nearrow \Lambda} \varkappa_{\alpha}(\lambda, \Lambda) P_{\Lambda}(x ; \alpha),
$$

where the multiplicities $\varkappa_{\alpha}(\lambda, \Lambda)$ are given by an explicit formula

$$
\varkappa_{\alpha}(\lambda, \Lambda)=\prod_{b \in v e r} \frac{(a(b) \alpha+l(b)+2)(a(b) \alpha+l(b)+1)}{((a(b)+1) \alpha+l(b)+1)(a(b) \alpha+l(b)+1)} .
$$


Here $b$ runs over all boxes in the $j$-th column ver of the diagram $\lambda$, provided that the new box $b_{0}=\Lambda \backslash \lambda$ belongs to the $j$-th column of $\Lambda$. The number $a(i, j)=\lambda_{i}-j$ is called the arm length, and $l(i, j)=\lambda_{j}^{\prime}-i$ is the leg length of a box $b=(i, j)$ in the diagram $\lambda$.

We define a generalization $\operatorname{dim}_{\alpha} \lambda$ of the dimension function $\operatorname{dim} \lambda$ by the recurrent formula

$$
\operatorname{dim}_{\alpha} \Lambda=\sum_{\lambda: \lambda \nearrow \Lambda} \varkappa_{\alpha}(\lambda, \Lambda) \operatorname{dim}_{\alpha} \lambda
$$

along with the initial condition $\operatorname{dim}_{\alpha}(\varnothing)=1$. If $\alpha=1$, the multiplicities are trivial, $\varkappa_{1}(\lambda, \Lambda) \equiv 1$, and $\operatorname{dim}_{1} \lambda=\operatorname{dim} \lambda$ is the ordinary dimension function.

The following hook-type formula for the dimension $\operatorname{dim}_{\alpha} \lambda,|\lambda|=n$, was found by R. Stanley [10]:

$$
\operatorname{dim}_{\alpha} \lambda=n ! \alpha^{n} \prod_{b \in \lambda}((a(b)+1) \alpha+l(b))^{-1} .
$$

We shall derive (6.4) as a particular case of Lemma 2.6.

To this end, we denote by $D_{\alpha}$ the dilation transform $D_{\alpha}(u, v)=(u, v \alpha),(u, v) \in \mathbb{R}^{2}$, and we consider the image $\Lambda^{\alpha}=D_{\alpha}(\Lambda)$ of a Young diagram $\Lambda$ upon this dilation. Note that the $\alpha$-content $c_{\alpha}(u, v)=v \alpha-u$ of a point $(u, v)$ coincides with the ordinary content $c(u, v \alpha)$ of the corresponding point $D_{\alpha}(u, v)$. We denote by

$$
x_{1}(\alpha)<y_{1}(\alpha)<x_{2}(\alpha)<\ldots<x_{d-1}(\alpha)<y_{d-1}(\alpha)<x_{d}(\alpha)
$$

the pair of interlacing sequences corresponding to the dilated shape $\Lambda^{\alpha}$. In other words, (6.5) is the sequence of $\alpha$-contents of corner points of the initial Young diagram $\Lambda$. The center of the pair (6.5) is trivial, $c(\alpha)=0$, and its area is $A(\alpha)=\alpha|\Lambda|$.

(6.6) Definition. Let $\Lambda$ be a Young diagram, $\lambda \nearrow \Lambda$, and assume that $\alpha>0$. By (6.3), the numbers $\varkappa_{\alpha}(\lambda, \Lambda) \operatorname{dim}_{\alpha} \lambda / \operatorname{dim}_{\alpha} \Lambda$ can be regarded as probabilities. We call them $\alpha$-co-transition probabilities of the diagram $\Lambda$.

(6.7) Theorem. Assume that a Young diagram $\lambda$ is obtained from $\Lambda \in \mathbb{Y}_{n}$ by erasing a box $b_{0}$ with the $\alpha$-content $y_{k}(\alpha)$ (more precisely, this is the $\alpha$-content of the south-east corner point of the box). Then

$$
\begin{aligned}
\frac{\varkappa_{\alpha}(\lambda, \Lambda) \operatorname{dim}_{\alpha} \lambda}{\operatorname{dim}_{\alpha} \Lambda} & =\frac{\left(x_{d}(\alpha)-y_{k}(\alpha)\right)\left(y_{k}(\alpha)-x_{1}(\alpha)\right)}{A(\alpha)} \times \\
& \times \prod_{i=1}^{k-1} \frac{y_{k}(\alpha)-x_{i+1}(\alpha)}{y_{k}(\alpha)-y_{i}(\alpha)} \prod_{j=k+1}^{d-1} \frac{y_{k}(\alpha)-x_{j}(\alpha)}{y_{k}(\alpha)-y_{j}(\alpha)}
\end{aligned}
$$


i.e., the $\alpha$-co-transition probabilities of a Young diagram $\Lambda$ coincide with the corresponding co-transition probabilities of the associated pair of interlacing sequences (6.5).

Proof. The proof is very similar to that of Lemma 3.3. By the $\alpha$-hook formula (6.4),

$$
\frac{\operatorname{dim}_{\alpha} \lambda}{\operatorname{dim}_{\alpha} \Lambda}=\frac{1}{n} \prod_{b \in v e r} \frac{(a(b)+1) \alpha+l(b)+1}{(a(b)+1) \alpha+l(b)} \prod_{b \in h o r} \frac{(a(b)+2) \alpha+l(b)}{(a(b)+1) \alpha+l(b)},
$$

where ver denotes the column of $\lambda$ above the new box $b_{0}=\Lambda \backslash \lambda$, hor is the row of $\lambda$ to the left of $b_{0}$, and all arm- and leg-lengths are taken with respect to the smaller diagram $\lambda$. We also took into account the $\alpha$-hook $h_{\alpha}\left(b_{0}\right)=((a(b)+1) \alpha+l(b))=\alpha$ of the box $b_{0}$.

Combining this with the multiplicity formula (6.2), we derive that

$$
\frac{\varkappa_{\alpha}(\lambda, \Lambda) \operatorname{dim}_{\alpha} \lambda}{\operatorname{dim}_{\alpha} \Lambda}=\frac{1}{n} \prod_{b \in v e r} \frac{a(b) \alpha+l(b)+2}{a(b) \alpha+l(b)+1} \prod_{b \in h o r} \frac{(a(b)+2) \alpha+l(b)}{(a(b)+1) \alpha+l(b)} .
$$

As we already did it in the proof of Lemma 3.3, consider the intersection $I$ of ver with a block of equal rows of the diagram $\lambda$. Observe that the arm lengths along $I$ are constant, and that the leg lengths increase by 1 . Assuming that the rows of $I$ correspond to the interval $\left(y_{j}(\alpha), x_{j+1}(\alpha)\right)$, we obtain that

$$
\prod_{I} \frac{a(b) \alpha+l(b)+2}{a(b) \alpha+l(b)+1}=\frac{x_{j+1}(\alpha)-y_{k}(\alpha)}{y_{j}(\alpha)-y_{k}(\alpha)}, \quad \text { if } j>k,
$$

and

$$
\prod_{I} \frac{a(b) \alpha+l(b)+2}{a(b) \alpha+l(b)+1}=x_{k+1}(\alpha)-y_{k}(\alpha), \quad \text { if } j=k .
$$

For a part $I$ of the row hor corresponding to the interval $\left(x_{i}(\alpha), y_{i}(\alpha)\right)$ we see that

$$
\prod_{I} \frac{(a(b)+2) \alpha+l(b)}{(a(b)+1) \alpha+l(b)}=\frac{y_{k}(\alpha)-x_{i}(\alpha)}{y_{k}(\alpha)-y_{i}(\alpha)}, \quad \text { if } i<k,
$$

and

$$
\prod_{I} \frac{(a(b)+2) \alpha+l(b)}{(a(b)+1) \alpha+l(b)}=\frac{y_{k}(\alpha)-x_{k}(\alpha)}{\alpha}, \quad \text { if } i=k .
$$

Since $A(\alpha)=n \alpha,(6.8)$ follows from (6.9) and the Theorem is proved.

In the course of the proof of the Theorem we employed the $\alpha$-hook formula (6.4) as a definition of the $\alpha$-dimension function. We did not use any connection with the recurrence formula (6.3). On the contrary, the hook formula (6.4) is actually a direct 
corollary of Theorem 6.7 and Lemma 2.6. In fact, Lemma 2.6 implies that the function defined by (6.4) satisfies the recurrence relation (6.3), hence the two definitions are equivalent.

(6.10) Corollary (Stanley, [10]). There is a hook formula

$$
\operatorname{dim}_{\alpha} \lambda=n ! \alpha^{n} \prod_{b \in \lambda}((a(b)+1) \alpha+l(b))^{-1}
$$

for the function $\operatorname{dim}_{\alpha} \lambda$ defined by (6.3).

7. The Plancherel growth process for anisotropic Young diagrams. In the previous Section we considered a system of multiplicities $\varkappa_{\alpha}(\lambda, \Lambda)$ for the edges of the Young graph, depending on a parameter $\alpha>0$. The multiplicities determine via the formula (6.3) a system of co-transition probabilities. By Theorem 6.7, these probabilities

$$
q_{\alpha}(\lambda, \Lambda)=\frac{\varkappa_{\alpha}(\lambda, \Lambda) \operatorname{dim}_{\alpha} \lambda}{\operatorname{dim}_{\alpha} \Lambda}, \quad \lambda: \lambda \nearrow \Lambda
$$

also arise from the general Definition 2.8, if one replaces every Young diagram $\Lambda$ by its dilated version $\Lambda^{\alpha}$. Presently, we want to describe a distinguished Markov chain on the Young lattice, which is central with respect to the co-transition probabilities (7.1). It will be a generalization of the Plancherel growth process for dilated (or anisotropic) Young diagrams.

(7.2) Lemma. Consider a function $\varphi: \mathbb{Y} \rightarrow \mathbb{R}$ defined as

$$
\varphi(\lambda)=\prod_{b \in \lambda}(a(b) \alpha+l(b)+1)^{-1} .
$$

Then

$$
\frac{\varkappa_{\alpha}(\lambda, \Lambda) \varphi(\Lambda)}{\varphi(\lambda)}=\prod_{i=1}^{k-1} \frac{x_{k}(\alpha)-y_{i}(\alpha)}{x_{k}(\alpha)-x_{i}(\alpha)} \prod_{j=k+1}^{d} \frac{x_{k}(\alpha)-y_{j-1}(\alpha)}{x_{k}(\alpha)-x_{j}(\alpha)}
$$

for every Young diagram $\lambda$.

Proof. It follows from the multiplicity formula (6.2) that

$$
\frac{\varkappa_{\alpha}(\lambda, \Lambda) \varphi(\Lambda)}{\varphi(\lambda)}=\prod_{b \in v e r} \frac{(a(b)+1) \alpha+l(b)}{(a(b)+1) \alpha+l(b)+1} \prod_{b \in h o r} \frac{a(b) \alpha+l(b)+1}{(a(b)+1) \alpha+l(b)+1}
$$

where hor and ver were defined in the proof of Theorem 6.7. 
Let us denote by

$$
x_{1}(\alpha)<y_{1}(\alpha)<x_{2}(\alpha)<\ldots<x_{d-1}(\alpha)<y_{d-1}(\alpha)<x_{d}(\alpha)
$$

the interlacing sequences corresponding to the dilated shape $\lambda^{\alpha}$, and assume that the $\alpha$-content of (the north-west corner point of) the new box $b_{0}=\Lambda \backslash \lambda$ is $x_{k}(\alpha)$.

Proceeding as in the proof of Lemma 4.3, we split the column ver into blocks of boxes with equal arm lengths. The $\alpha$-hooks $(a(b)+1) \alpha+l(b)$ in such a block $I$ vary from $y_{j-1}(\alpha)-x_{k}(\alpha)$ to $x_{j}(\alpha)-x_{k}(\alpha)-1$, for some $j>k$. Hence,

$$
\prod_{b \in I} \frac{(a(b)+1) \alpha+l(b)}{(a(b)+1) \alpha+l(b)+1}=\frac{x_{k}(\alpha)-y_{j-1}(\alpha)}{x_{k}(\alpha)-x_{j}(\alpha)}
$$

and

$$
\prod_{b \in v e r} \frac{(a(b)+1) \alpha+l(b)}{(a(b)+1) \alpha+l(b)+1}=\prod_{j=k+1}^{d} \frac{x_{k}(\alpha)-y_{j-1}(\alpha)}{x_{k}(\alpha)-x_{j}(\alpha)}
$$

Likewise,

$$
\prod_{b \in h o r} \frac{a(b) \alpha+l(b)+1}{(a(b)+1) \alpha+l(b)+1}=\prod_{i=1}^{k-1} \frac{x_{k}(\alpha)-y_{i}(\alpha)}{x_{k}(\alpha)-x_{i}(\alpha)},
$$

and the formula (7.4) follows.

(7.5) Theorem. The numbers $p_{\alpha}(\lambda, \Lambda)=\varkappa_{\alpha}(\lambda, \Lambda) \varphi(\Lambda) / \varphi(\lambda), \lambda \nearrow \Lambda$, form a system of transition probabilities for a Markov chain on the Young graph, central with respect to the edge multiplicities (6.2).

Proof. By (7.4) and Lemma 2.2, the numbers $p_{\alpha}(\lambda, \Lambda)$ form, for every fixed Young diagram $\lambda$, a probability distribution. The probability to reach a given Young diagram $\Lambda \in \mathbb{Y}_{n}$ by a particular Young tableaux $t=\left(\varnothing=\lambda_{0} \subset \lambda_{1} \subset \ldots \subset \lambda_{n}=\Lambda\right)$ is easily seen to be

$$
P_{\alpha}(t)=\varphi(\Lambda) \prod_{k=1}^{n} \varkappa_{\alpha}\left(\lambda_{k-1}, \lambda_{k}\right)
$$

hence the co-transition probabilities are $q(\lambda, \Lambda)=\varkappa_{\alpha}(\lambda, \Lambda) \operatorname{dim}_{\alpha} \lambda / \operatorname{dim}_{\alpha} \Lambda$, for every $\Lambda$, and the chain is central.

In complete analogy with Corollary 5.6 we derive from (7.4) and Corollary 5.5 that the numbers

$$
p_{u, v}(\lambda, \Lambda)=\frac{\left(c_{\alpha}(b)+u\right)\left(c_{\alpha}(b)+v\right)}{n \alpha+u v} \frac{\varkappa_{\alpha}(\lambda, \Lambda) \varphi(\Lambda)}{\varphi(\lambda)}
$$


sum up to unity. If all of these numbers are positive (e.g., if $v=\bar{u}$ ) we can think of a Markov chain on the Young lattice with transition probabilities (7.6).

(7.7) Corollary. The Markov chain with the transition probabilities (7.6) is central with respect to co-transition probabilities (7.1).

Proof. For every Young tableaux $\varnothing=\lambda_{0} \subset \lambda_{1} \subset \ldots \subset \lambda_{n}=\Lambda$, the product

$$
\prod_{k=1}^{n} \frac{p_{u, v}\left(\lambda_{k-1}, \lambda_{k}\right)}{\varkappa_{\alpha}\left(\lambda_{k-1}, \lambda_{k}\right)}=\frac{\varphi(\Lambda)}{u v(u v+\alpha) \ldots(u v+(n-1) \alpha)} \prod_{b \in \lambda}\left(c_{\alpha}(b)+u\right)\left(c_{\alpha}(b)+v\right)
$$

depends on the final Young diagram $\Lambda$ only. This means that the Markov chain is central.

\section{REFERENCES}

1. S. V. Kerov, A q-analog of the Hook Walk Algorithm for Random Young Tableaux, J. of Algebraic Combinatorics 2 (1993), 383 - 396.

2. S. V. Kerov, The Boundary of Young Lattice and Random Young Tableaux, DIMACS Series in Discrete Mathematics and Theoretical Computer Science 24 (1996), 133-158.

3. S. V. Kerov, Asymptotics of root separation of orthogonal polynomials (in Russian), Algebra and Analysis 5-5 (1993), 68-86.

4. S. V. Kerov, Transition Probabilities of continuous Young diagrams, and the Markov Moment Problem, Funct. Analysis and its Applications 27-2 (1993), 32 - 49.

5. S. V. Kerov, Interlacing Measures, Preprint LaBRI 1116 (1996), LaBRI, Université de Bordeaux-I, $1-53$.

6. S. V. Kerov, G. I. Olshanski, A. M. Vershik, Harmonic Analysis on the Infinite Symmetric Group, Comptes Rend. Acad. Sci. Paris 316 (1993), 773-778.

7. S. V. Kerov, A. M. Vershik, The Grothendieck Group of the Infinite Symmetric Group and Symmetric Functions with the Elements of the $K_{0}$-functor theory of AF-algebras, Representation of Lie groups and related topics (A. M. Vershik and D. P. Zhelobenko, eds.), Adv. Stud. Contemp. Math., vol. 7, Gordon and Breach, 1990, pp. 36-114.

8. A. N. Kirillov, Lagrange Identity and the Hook Formula, Zapiski Nauchn. Sem. LOMI 172 (1989), $78-87$.

9. I. G. Macdonald, Symmetric functions and Hall polynomials, 2nd edition, Oxford University Press, 1995.

10. R. P. Stanley, Some Combinatorial Properties of Jack Symmetric Functions, Adv. Math. 77 (1989), 76-115.

11. A. M. Vershik, The Hook Formula and Related Identities, Zapiski Nauchn. Sem. LOMI 172 (1989), $3-20$.

12. A. M. Vershik A., S. V. Kerov, The asymptotics of maximal and typical dimensions of irreducible representations of the symmetric group (in Russian), Functional Analysis and its Applications 19-1 (1985), 25-36.

Steklov Math. Institute (POMi), Fontanka 27, St.Petersburg, 191011, Russia

E-mail address: kerov@pdmi.ras.ru 